\title{
Marine Engine Room Alarm Monitoring System
}

Fault Detection and Monitoring Technique via PLCs and SCADA-based System

\author{
Isaac Tawiah, Usman Ashraf, Yinglei Song, Aleena Akhtar \\ School of Electronics and Information Science, \\ Jiangsu University of Science and Technology, \\ Zhenjiang, 212003, China
}

\begin{abstract}
Alarms affect operations in most part of the ship. Their impact on modern Engine Control Room operations is no less significant. The state of an alarm system serves as an indication of the extent to which the ship's operations are under management control. Thus, the design of efficient and reliable alarm monitoring system is vital for safe and sound operations. Although several design techniques have been proposed, all the proposed design methods employ sophisticated and expensive approaches in resolving alarm issues. In this paper, a cheap, yet reliable and efficient alarm design method for engine room device monitoring is presented. The design method employs PLCs and SCADA-based system and adopts certain basic design requirements of alarm monitoring system presented in literary works. Reasons for such a design method are highlighted, and the programming platforms for the design are given. The strengths and weaknesses of some design methods presented in some published works are reported and solutions to such problems are proposed. The proposed design technique, including fault diagnostic algorithm, have been subjected to real-time online testing at the shipyard, specifically Changjiang Waterway Bureau, China (ship name-Ning Dao 501). The testing results proved that this design technique is reliable, efficient and effective for online engine control room device monitoring.
\end{abstract}

Keywords-Alarm monitoring system; engine control room; OPC communication; PLCs; SCADA systems

\section{INTRODUCTION}

Reports indicate that alarm monitoring issues are the biggest single issue affecting safety in modern engine control rooms [1], [2], [3], [4]. Reported issues include distractions from nuisance alarms, confused and unclear alarm messages, inappropriate use of alarms to convey non-critical information, long standing alarm lists, and difficulty in alarm interpretations. Moreover, several works have been proposed to mitigate these issues. In [5], Zaghloul presented a practical design of an open architectural ship alarm control and monitoring systems via SCADA system. In Zaghloul's proposal, a collection of standard software (Human Machine Interface) is used to provide a SCADA central host of all the statuses of the field machinery. The shortfall of this design method is that, it only performs monitoring purposes, and ignores basic error detection algorithms. In resolving such design issues, Lee et al. [6], [10], [11] proposed basic design requirements of alarm systems, whereas [7], [8] propose fuzzy logic algorithms to tackle fault detection issues. A SCADA based system for monitoring a central heating and power plant using Siemens equipment and software "Process Control System 7" (PCS7) was proposed in [9]. This design technique employs redundant servers and web-based applications via OPC and web servers to provide remote actions and uninterrupted monitoring. Aside the sophisticated and expensive nature of the method proposed in [9], it is incapable of reporting errors that incurred during device malfunctions. Problems of unreliable communication resulting from complex cabling have also been reported in [12]. In resolving these problems, D'ana [13] discusses a communication based model over GSM network to remotely monitor and control PLC based processes. The design approach is built on industrial PLCs, where each PLC is integrated with processors capable of connecting to a network via GSM modem. Dana's intent was to provide system users with a backup communication mechanism in case of a network failure. Even though capitally intensive, the idea proved to achieve the same functionality as Profinet but at a higher bandwidth (10/100 Mbps). Wang et al. [14], also presented a communication design method based on CAN bus and RS485. All the above reported solutions employ sophisticated and expensive approaches in resolving the above highlighted issues.

In this paper, the basic design requirements of alarm monitoring system presented in [6] are adopted, and the communication methods of [13] are used to resolve these issues. However, instead of using the more sophisticated technique proposed in [13], the TCP/IP Ethernet based network, via PLC is used for PC communication. In addition, the fault diagnostic algorithm employed in this paper is different from those presented in literature [7]. Most of the methods reviewed in [7] employ two or more sensors to monitor a single parameter (e.g. Temperature). However, while cost is always an important factor, process and personnel safety are also important. Specifying multiple sensors to gain comparative readings, with the aim of obtaining a voting logic leads to added capital cost, added cost of spare sensors to maintain capability while recalibrating units, and perhaps even additional staff personnel to perform calibrations and periodic checks [15]. Thus, with the view of cutting down cost, an error control algorithm is attached to just a single input sensor. From an electrical perspective, it is also appropriate to protect circuits in order to ensure safe operation of the PLCs and field I/O devices. The protection of circuits is often done by fusing each $\mathrm{I} / \mathrm{O}$ module with a single fuse [16], [17]. In this paper, the technique where each I/O point is fused is used to guarantee that one fault only disrupts the affected point [18].

\section{OVERVIEW}

This paper presents a design method for real-time monitoring of engine room alarm systems, using PLCs and SCADAbase system. Siemens S7-1200 Series PLC (CPU 1214C DC/DC RLy) is used. Whilst this paper is targeting this type of PLCs, the approach used here, can be transferred to other 
Vendor and System types. Communication between PLC and $\mathrm{PC}$ is achieved through Siemens Totally Integrated Automation (TIA) portal software, via Ethernet TCP/IP protocol. This allows simulations to be performed through Ethernet communication with the PC, even before real-time test is carried out in the control room. OLE for Process Control (OPC) is used as a communication server between TIA and the SCADA GUI (NI LabVIEW) software. The data collected is the parameters of the various monitored field machinery. Thirty-Five monitored functions, under five main parameters were considered, including Temperature, Pressure, Voltage/Current, Speed, and Depth of Liquids. Data acquisition was made possible through sensors connected to the input channels of the PLC modules. The designed alarm monitoring system, including the fault diagnostic algorithm was subjected to real-time online testing at the shipyard, specifically Changjiang Waterway Bureau, China (ship name-Ning Dao 501).

Fig. 1a. shows the screenshot of the deployed PLC, showing the signal modules (from slot 102A1 to 102A3), the CPU (slot 102A4), and the signal modules (from slots 102A5 to 102A8). This series of PLC utilizes a maximum of three communication modules for serial communication, and up to eight signal modules for input/output expansion. In this paper, all the three communication modules are utilized, as labeled 102A1 to $102 \mathrm{~A} 3$ in Fig. 2. Four of the eight available signal modules are used. This constitutes the labels 102A5 to 102A8. Fig. 3 shows the electrical network of the PLC workstation. The workstation constitutes both the CPU and all other modules connected to it. The series of communication modules used is the CM1241 series with port number 1RS485.

The Uninterrupted Power Supply (UPS) used here is a separate standalone, and thus additional pieces of power supplies are needed to convert the correct voltages to power various devices. In the case of the deployed PLC, the maximum allowable input voltage is $24 \mathrm{~V}$ DC. A voltage of $220 \mathrm{~V} \mathrm{AC}$ of $2.5 \mathrm{~A}, 50 / 60 \mathrm{~Hz}$ is stepped-down to $24 \mathrm{~V} \mathrm{DC}$ at $8.8 \mathrm{~A}$ to power the PLC. As seen from Fig. 4, each power supply connection is connected by a $2 \mathrm{~A}$ fuse for circuit protection. The industrial $\mathrm{PC}$, the monitor, the field sensors, all have their own operating voltages. A $220 \mathrm{~V}$ of $1 \mathrm{KW}$ AC power source supplies power to other secondary power supplies, both in the engine room and in the ship's cabin across a $2 \times 1.5 \mathrm{~mm}$ and a $2 \times 2.5 \mathrm{~mm}$ wires.

\section{DeSIGN Methodology}

The main idea is to design a reliable and effectively cheap alarm monitoring and fault detection system. The general architectural design employed in this work is summed up in the diagram of Fig. 5. Sensors attached to field devices receive and transmit data to the PLC inputs via communication cables. Unlike the various design methods presented in the literature where two or more sensors are used to access a single input parameter, the proposed technique employs a single sensor for parameter assessments. Data-flow from the PLC to PC proceeds through TCP/IP communication. Within the Siemens TIA portal software, an efficient error control algorithm is developed to provide alarm signals of the malfunctions. This malfunctions and all other signals are made accessible to the operator on a GUI through OPC server station. In summary, the following contributions are made: the technique serves two

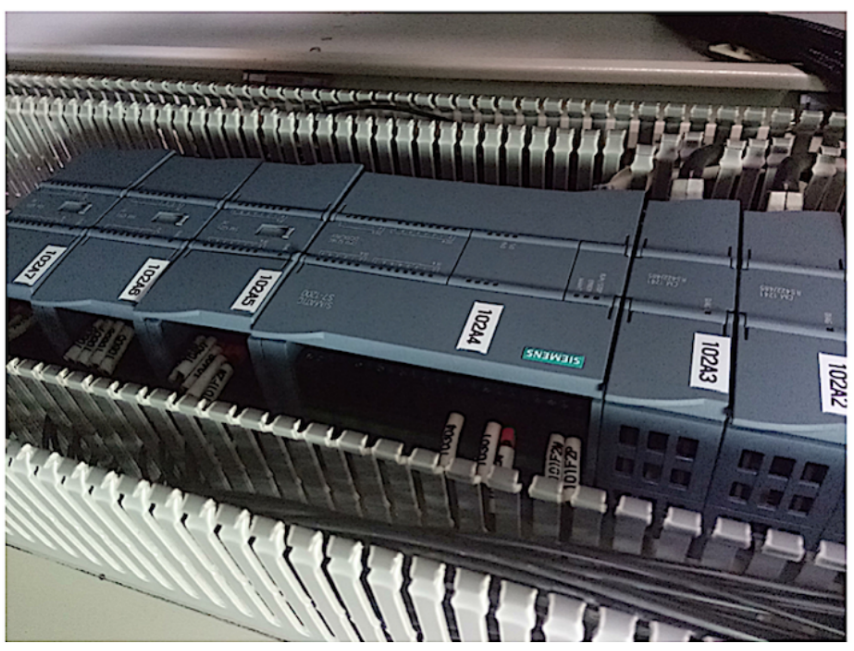

a. Deployed PLC

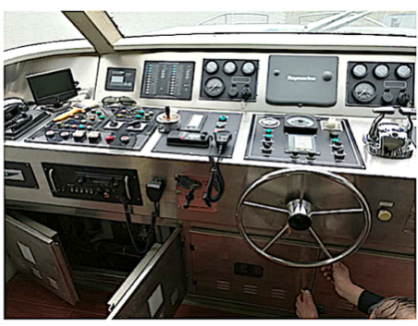

b. Control Room

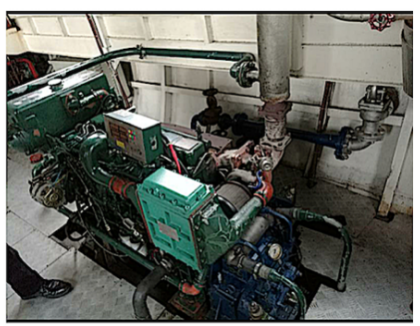

c. Main Engine
Fig. 1. Screenshots of the deployed PLC workstation, and some Engine room machinery.

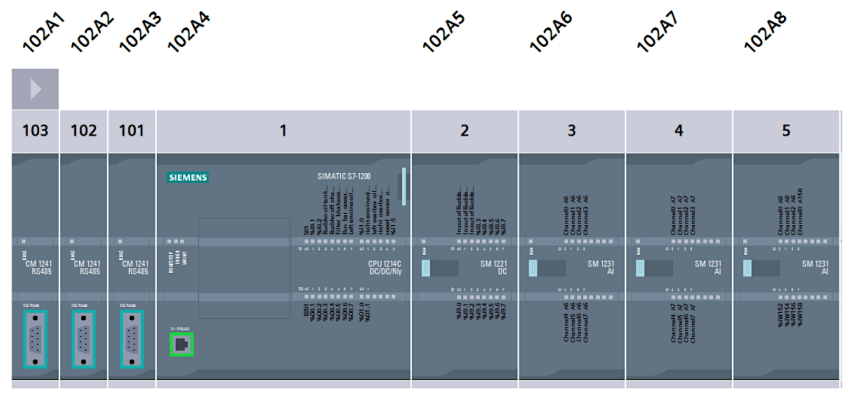

Fig. 2. PLC 1214C workstation.

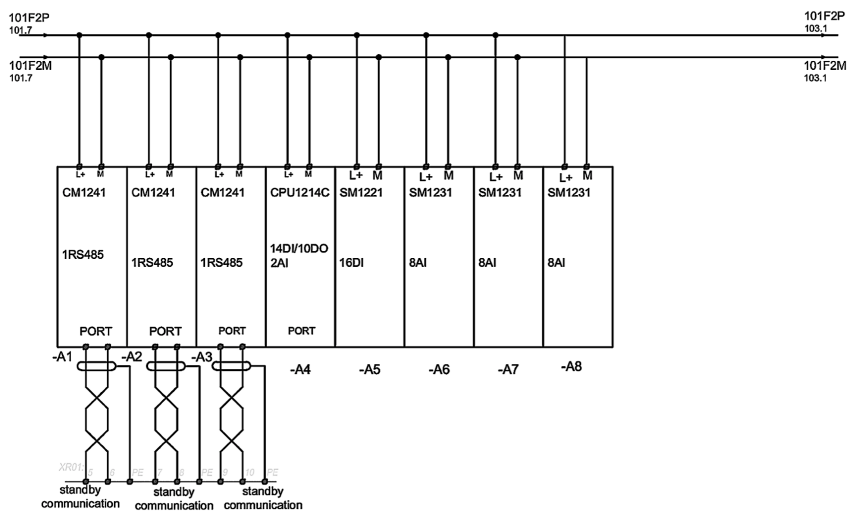

Fig. 3. CPU 1214C workstation wiring diagram. 


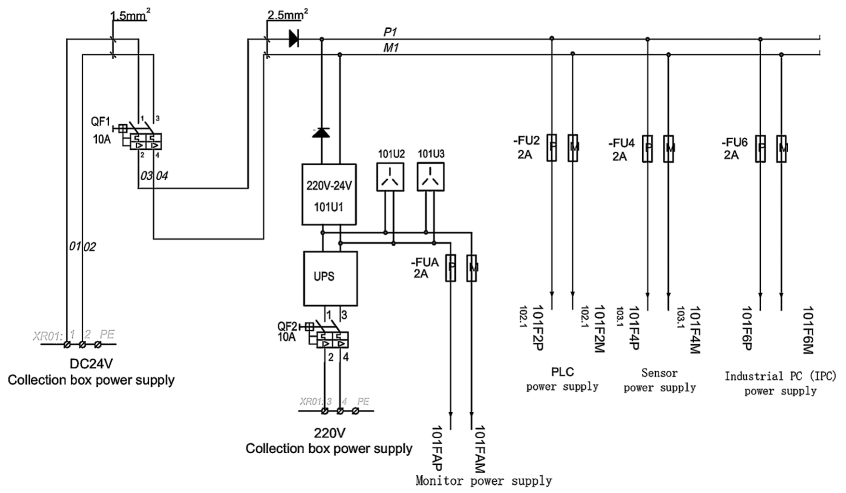

Fig. 4. Schematic of the system power supply.

purposes - both monitoring and fault diagnosis; it is capitally cheap, yet a reliable technique; all the communication proceeds through TCP/IP, through Ethernet cables; and it is able to store alarm record list for future fault identification. The details of the design technique are presented in the subsequent sections.

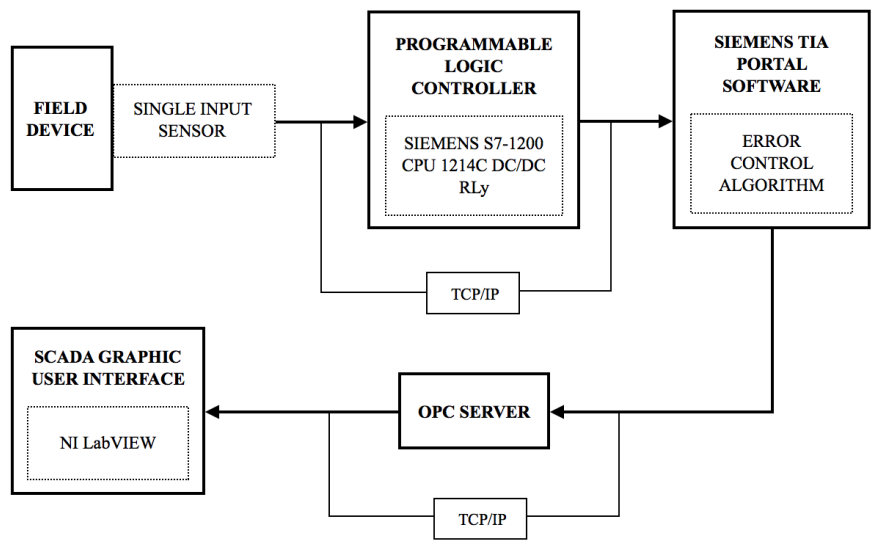

Fig. 5. General architectural design flowchart.

\section{Monitoring Functions}

\section{A. Analog Functions}

Analog sensors are connected to the PLC via analog modules without additional amplifiers. The input modules SM 1231 AI from slot $102 \mathrm{~A} 6$ to $102 \mathrm{~A} 8$ (see Fig. 2) are used for the input analog sensors. Each of the modules has eight channels counted from 0 to 7 , with a selected current or voltage range of either $4 \mathrm{~mA}-20 \mathrm{~mA}$ or $-10 \mathrm{~V}$ to $10 \mathrm{~V}$ respectively. Each channel can store integer data ranging from 0 to 32767 , however, all integer limits in this work are from 0 to 27648 . In total, nineteen analog sensors are monitored (see Fig. 6 to 8), including voltages, currents, temperatures, and pressures. In cases where levels of fluids are to be measured, the formula $p=\rho h g$ is used to convert the measured pressure value to height. As seen from Fig. 6 to 8 , each of the channels is protected by a $0.1 \mathrm{~A}$ fuse. Each of the input is labeled FUxx, where FUxx represents the fuse number. For instance, the analog input "Power station Voltage" is labeled FU01, with a $0.1 \mathrm{~A}$ fuse protection. This mode of identification provides easier access to faulty sensors, as this identification scheme is used in the alarm logical programming.

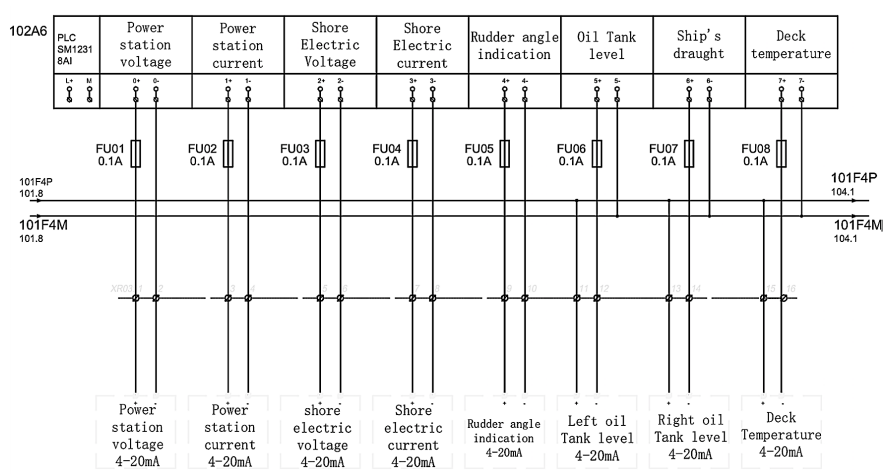

Fig. 6. Analog signals (signal module SM 1231, slot 102A6).

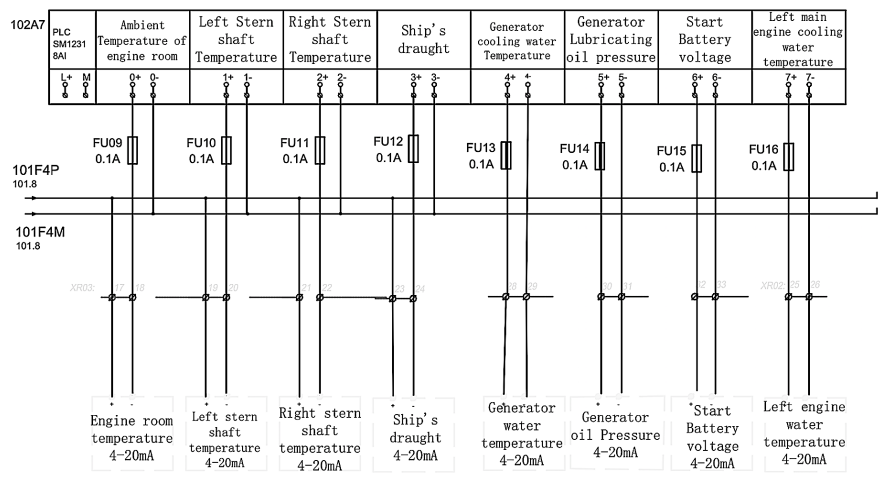

Fig. 7. Analog signals (signal module SM 1231, slot 102A7).

\section{B. Digital Functions}

Digital sensors are connected to the PLC via digital modules. The extensive range of digital modules allows the most suitable signal module to be selected in each case. Sixteen digital inputs are monitored in this work via an SM 1221 DC signal module. This module is located in the first slot of the four signal module, labeled 102A5. Similarly, it houses 16 channels ranging from channel 0 to 15 . Fig. 9 and 10 show the electric charts for the input digital sensors.

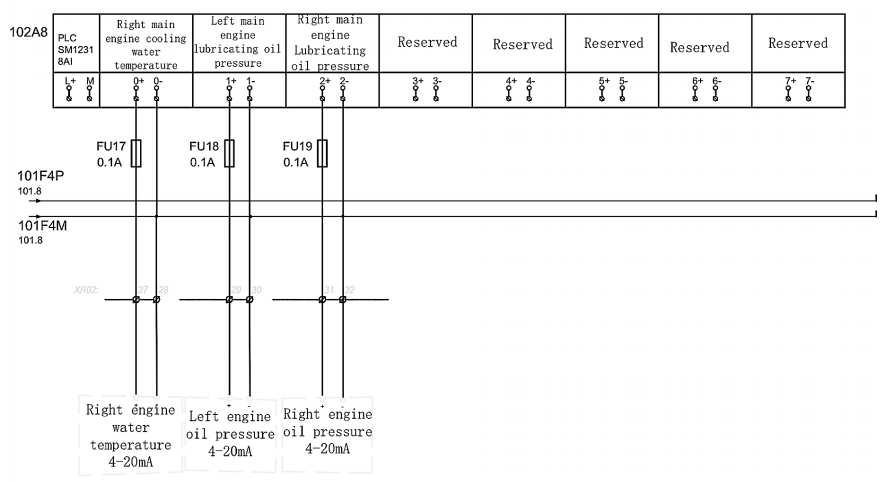

Fig. 8. Analog signals (signal module SM 1231, slot 102A8). 


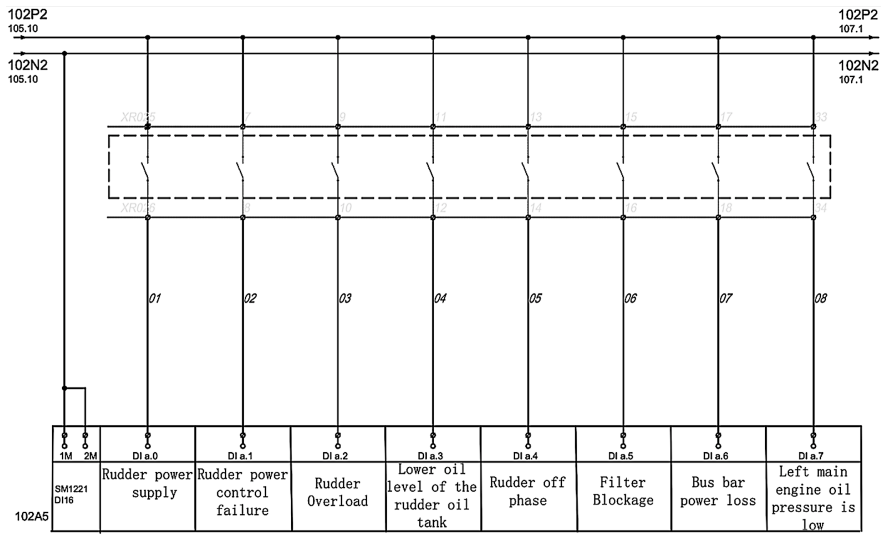

Fig. 9. Digital signals (signal module SM 1221, slot 102A5).

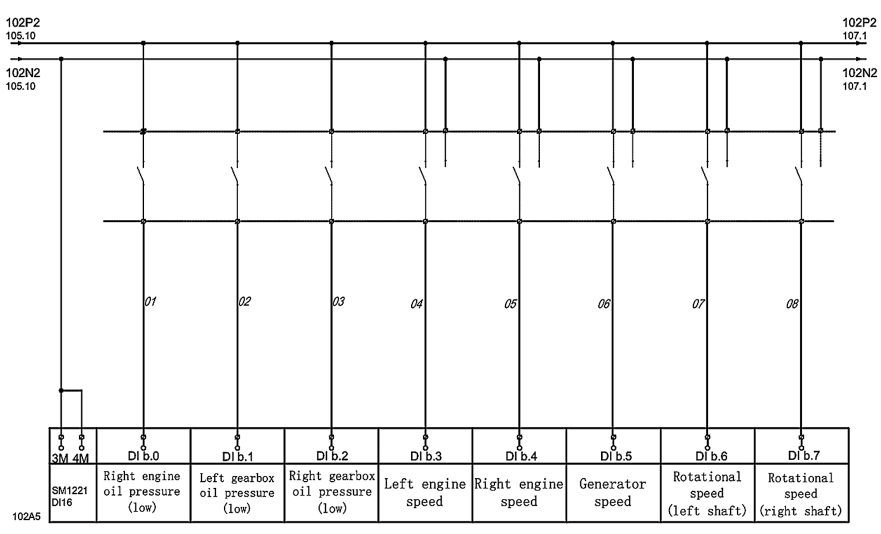

Fig. 10. Digital signals (signal module SM 1221, slot 102A5).

\section{EfFicient Fault Diagnosis Algorithm}

The design of fault diagnosis algorithms in control theory follows three main steps: Fault detection, isolation and recovery. This involves monitoring a system, identifying when a fault has occurred, and pinpointing the type of fault and its location. Generally, two main approaches come in a handy. One, is performing a direct pattern recognition of sensor readings that indicate a fault, and the other is analyzing the discrepancy between the sensor readings and expected values that have been calculated from some model. In the second case, the fault is said to be detected if the discrepancy or residual is above or below a certain threshold. Immediately the fault is detected, it has to be isolated and its location has to be displayed. The fault detection algorithm used in this paper follows the second approach. In other words, an error control logic is attached to all the input sensors, and the responses or outputs of the imposed conditions are displayed on the virtual innovation interface (LabVIEW). The algorithm was designed to be non-intrusive, in recognition that other existing SCADA systems often accommodate fragile timing constraints. The flow chart depicting the detailed blueprint of the logics is given in Fig. 11.

\section{SCADA DESIGN INTERFACE}

The SCADA design interface is divided into three major sections. The Indicator section consists of all the alarms, and limit displays. This includes the alarms that require quick

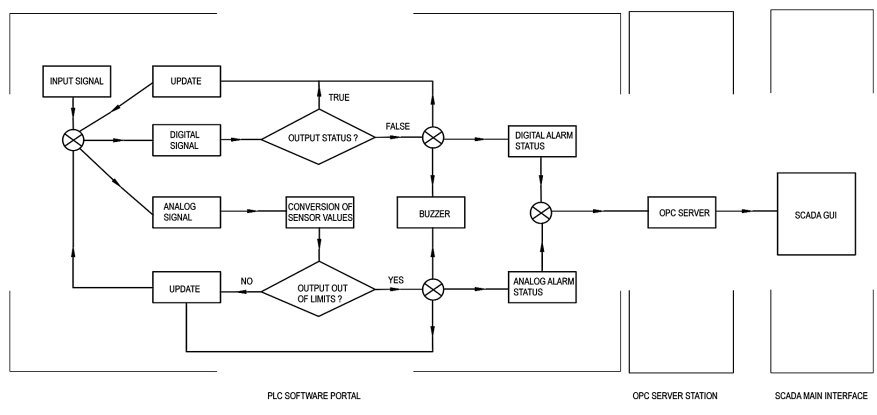

Fig. 11. Logic based alarm design algorithm.

response by the operator, and alarms that are frequently used. The Plant section produces a mimic display of the plant status. It also has an Error Report Section, or a segment and distributed architecture that reports failures and faults in real-time processing. The SCADA system is able to process new changes made in the PLC logics to improve signal validation, and alarm suppression. In other words, the display contains at least alarm status, current value, set-point values, and point identification. Input signals from sensors to the system (SCADA GUI) are put into limit checks, necessary engineering unit conversions (e.g. from Kelvin to degree celsius, from atm to Kpa, etc.), and signal validation to calculate the representative values from multiple sensor channels. In order to realize real-time processing, the signal validation is proceeded through simple averaging algorithms. The main purpose of designing alarm and control display is to maximize operator-machine interface visibility. Therefore, the alarm status display is color coded, and indicator buttons are put into different shapes, and are grouped by system and function (that is the analog and digital functions are separated by different Tabs).

\section{Programming Logics - AnAlog Signals}

\section{A. Monitoring - Voltage and Current}

We highlighted in Section IV-A the number of analog signals monitored in this project. In all, nineteen analog signals were being monitored. These signals can be grouped into five categories; as either Voltages, Currents, Pressures, Temperatures, or Levels of fluids. Details of the programming logics of each category is presented and analyzed in this section. The functional block diagram and the logical codes behind the blocks are shown for the monitored voltages/current ("power station") in the codes of Fig. 12. In all cases, the incoming voltage from the voltage sensors to the PLC input is an integer value which needs to be converted into a real value voltage. Internal $\mathrm{A} / \mathrm{D}$ converter and conversion factors are used to produce a real value output voltage, via predetermined temporary variables (see lines 5 to 12 of the codes). Based on the operating conditions of the field devices powered on by the supplied voltage, operating limits are imposed on the monitored voltages. The PLC channel voltage ranges from $10 \mathrm{~V}$ to $10 \mathrm{~V}$, thus all voltages are within the limits. Similar to the voltage signals, current monitoring proceeds through input current sensors. This input sensor detects the electric current, and then generates a signal proportional to that current. The input DC current appears at the input of the PLC as an integer value which is then converted into a real value output current. 
The imposed limits fall within the 4mA-20mA DC operational limits of the input channels of the analog signal module. The availability of functional block diagrams in the TIA portal software provides a genuine opportunity for the operator to monitor both the input and output values of the connected voltage/current sensors. This provides significant amount of information about the status of the monitored voltages/currents. The imposed limits on the outputs similarly serves as checks to avoid device damages. From the block diagram, it can be observed that the inputs are put into checks within "MIN_VAL" and "MAX_VAL". The tag name FUxx, where FUxx signifies the fuse number, provides easier identification of the monitored device. For instance, FU01_OUT and FU02_OUT indicate the output status of the voltage and current signals, respectively.

The output values and the status of the monitored signals are controlled by a Structured Control Language (SCL) codes behind the functional blocks. Each block is controlled by a different set of codes, with the results observed by the output tags. Within the SCL platform, the operator also gets access to the alarm status of the monitored functions. The maximum values, the minimum values, output and alarm status are all available within the SCL background, as seen from the far right of the voltage codes. Lines 1 to 12 of the codes entails the conversion factors employed in generating the actual output voltage. The parameters hash-tagged VOLTxx are temporary imposed variables within the SCL tags to temporary store the input variables from the voltage sensors. The output is then realized after the input conversion. In order to realize and make accessible of the minimum/maximum voltage values in the SCADA GUI, we store the temporary MIN_VAL_FUxx and MAX_VAL_FUxx in a permanent transferable memory. For instance, MIN_VAL_FU01 and MAX_VAL_FU01 are stored in the permanent real-value memory location MIN_FU01 and MAX_FU01, respectively. Inasmuch as the limits have been imposed to monitor the voltage, an error check criterion is also used to provide additional checks on the voltage surges (from lines 19 to 22). A conditional value of 0.01 is used as a limit check for the surges that may incur during high fluctuation in the voltages. During high voltage spikes, this error check is very significant.

\section{B. Monitoring - Temperature}

Temperature is one of the most widely measured parameters in the ship's engine room. The two most commonly used temperature sensors are the Resistance Temperature Detectors (RTDs) and the Thermocouple. The temperature sensors employed in the engine room, for this work, are the two sensors mentioned above. RTDs have internal resistance that changes with temperature in a predictable, linear way, and hence suitable for small temperature ranges, such as measuring the temperature of water. For wider temperature ranges, up to a couple of thousand degrees, the thermocouple was used. Thermocouple works based on the seebeck effect, where a small voltage is produced across a junction of two dissimilar metals when exposed to temperature gradient. Depending on the temperature characteristics of the field device whose temperature is to be monitored, different limits are imposed on different input sensors. For instance, the limits imposed on the Deck temperature is from -5 to 42 degrees celsius, which is a good range for monitoring environmental temperature. Explanations of the codes for the Deck Temperature is

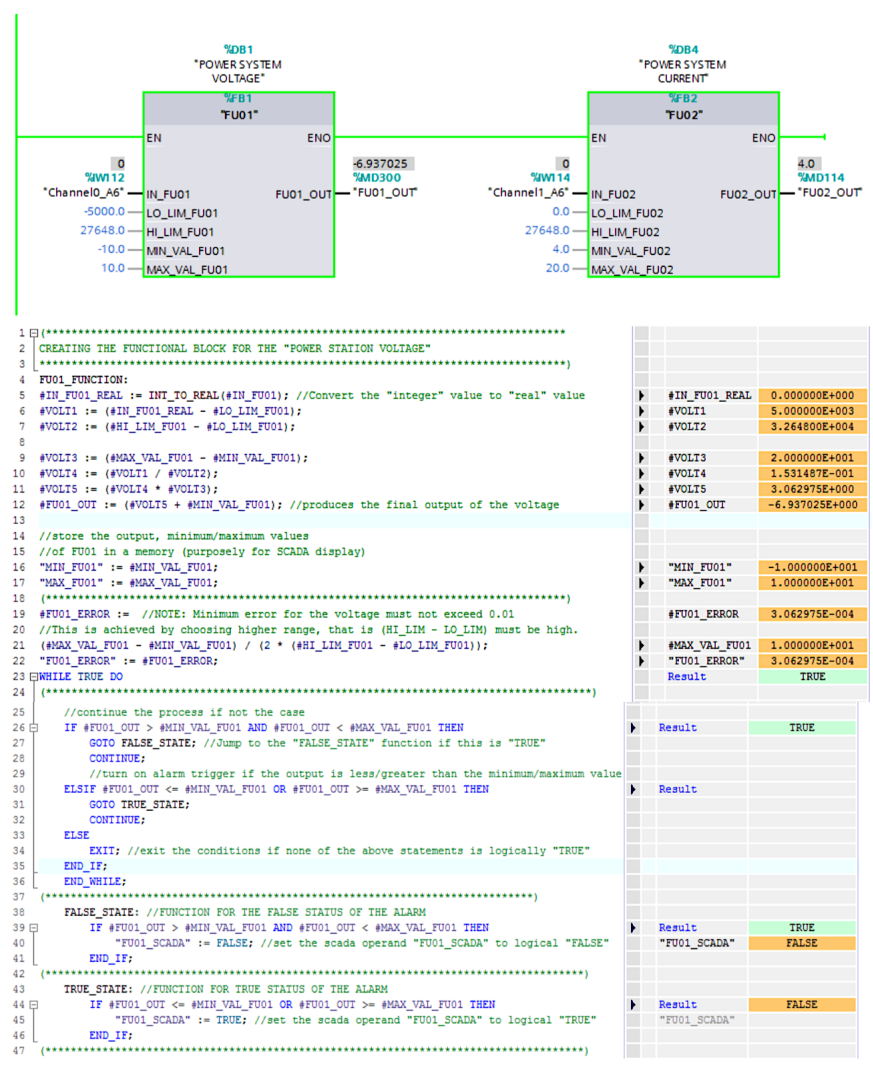

Fig. 12. Functional block diagram and SCL codes for Voltage/Current.

provided in Fig. 13. There are seven monitored temperatures in total, including Deck Temperature, Engine Room, Engine water, and Shaft Temperatures. Just as in the codes for all the other analog functions, lines 4 to 12 produce the real output temperature value, while lines 14 to 15 store the minimum/maximum limits in a permanent working memory. The output and limits are controlled by the lines 21 to 42 of the codes. These conditions are imposed for monitoring purposes. The FUxx_SCADA in all the codes is a bit memory location that indicate either logical TRUE or FALSE depending on the restrictions imposed on the output. For instance, the imposed limits on the deck temperature is between -5 to 36 degrees celsius. Thus FU08_SCADA indicates logical FALSE if the output falls within this range, otherwise TRUE. This is continuously updated as long as the field device and the alarm logics are operational. The speed of this repeated updates and data sequences is controlled by the PLC's internal clock counters and frequencies. The status of FU08_SCADA triggers the MS01 bit to act in a corresponding way. If MS01 is TRUE, then the signal triggers the BUZZER, BS01 to sound. The flexibility that comes with the TIA portal software makes it easier to monitor the statuses from both within the SCL, FBD programming platform as well as on the Ladder Logic platform. In this paper, all the analog statuses are linked up to a single alarm block (see Section IX). The alarm logics, as well as the functional block diagram used for monitoring the deck temperature is presented in Fig. 13. 

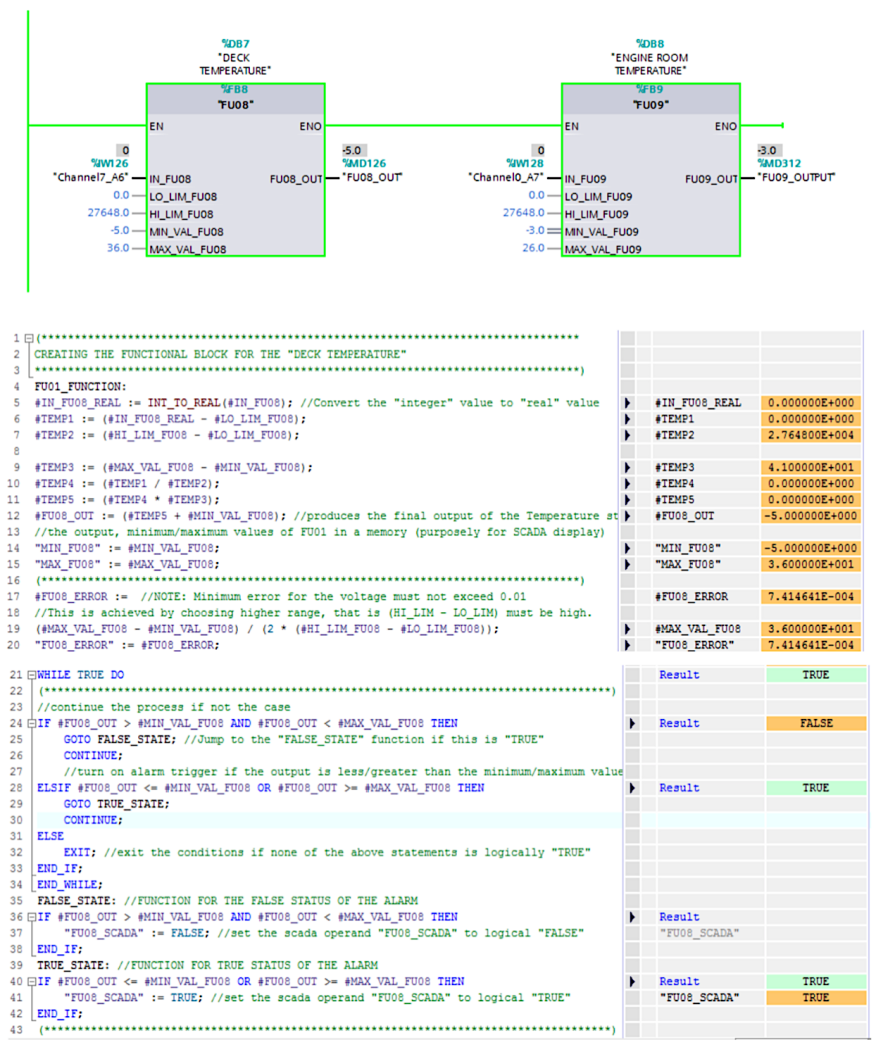

Fig. 13. Functional block diagram and SCL codes for monitoring temperature.

\section{Monitoring: Pressure and Level of Fluids}

Both pressure and fluid levels proceed through the use of pressure sensors, acting as $4-20 \mathrm{~mA}$ transducers. The logical codes for the pressure monitoring follows the same logics as that of the temperature. Three pressure sensors are monitored. This includes Engine oil pressures and generator oil pressure. Fluid levels were monitored using pressure sensors, by taking a continues pressure measurement from the bottom of the tank or point where the sensor's diaphragm is placed. Tanks in the engine room were vented to the atmosphere, and so pressure sensors were configured with a vent tube to correct for barometric pressure changes. Levels were calculated from pressure values using the simple relation $p=\rho h g$. Thus the pressure exerted by a column of oil in the tank is dependent solely on the specific gravity of the oil, the level of oil, and the gravitational acceleration. The input pressure sensor values continuously update, and the corresponding level calculated from the given formula above. The values of density, $\rho$ of oil and gravity, $g$ employed in the calculations were $830.0 \mathrm{~kg} / \mathrm{m}^{3}$ and $10.0 \mathrm{~m} / \mathrm{s}^{2}$, respectively. Similar to the case of pressure functions, Engine oil levels, and generator oil level were the monitoring level functions. The codes in Fig. 14 indicate the logics behind the Oil Level measurements. Input limits in the block diagrams are modifiable to the operating conditions.

\section{Programming Logics: Digital Signals}

Sixteen digital functions are monitored in this project. Ladder Logic Programming dominates, except where SCL had to be created to calculate Engine Speed. Digital sensors pass signals to the PLC input, which in turn is recorded in a bit
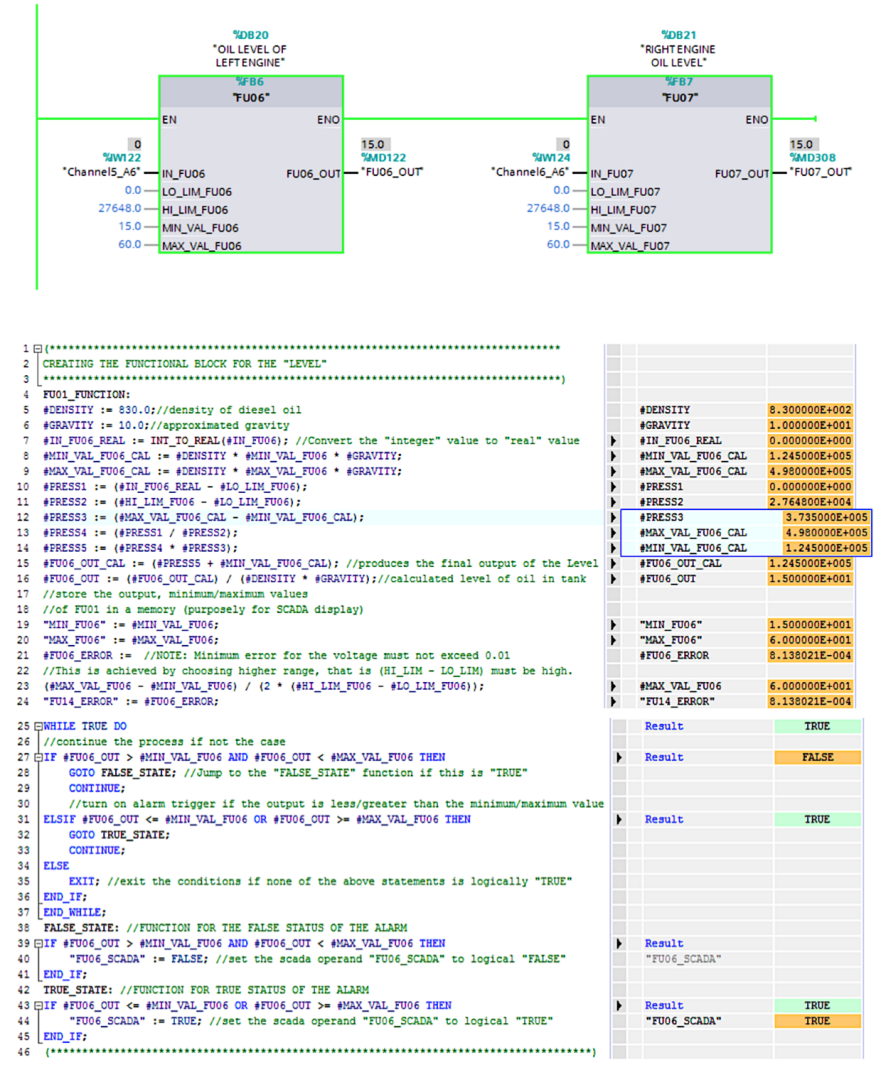

Fig. 14. Functional block diagram and SCL codes for monitoring pressure and level of fluids.

memory location as logical TRUE or FALSE. Output of the digital alarm block receives and acts on the status of the recorded logic. Just as all logics, boolean outputs are labeled FUxx_SCADA, where FUxx represents the Fuse label. Ladder logic for engine speed is presented in Fig. 15.

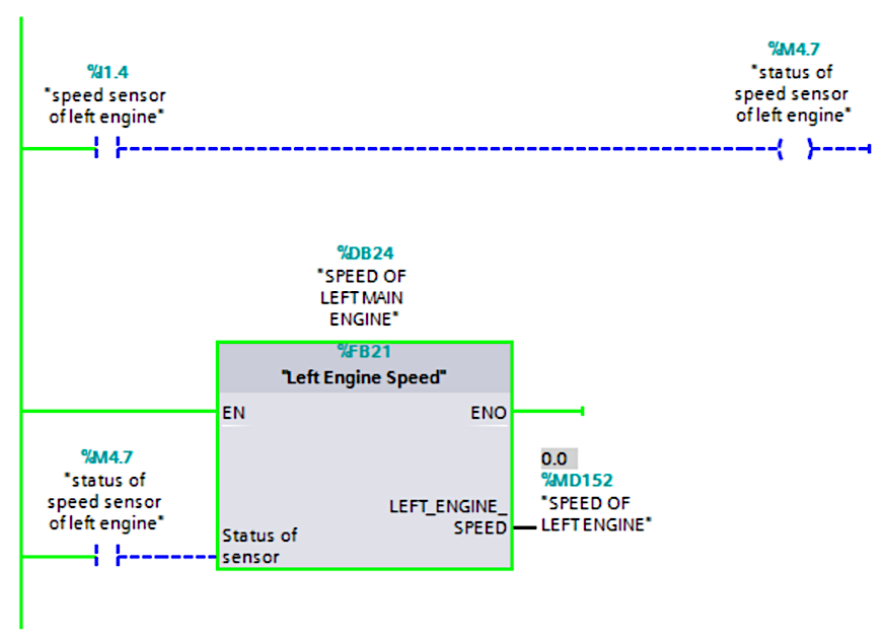

Fig. 15. Ladder logic and functional block diagram for the left engine speed.

\section{Programming Logics: Alarm}

The alarm architecture constitutes a muffler button and a buzzer, all connected to the input and output respectively, 
of the CPU in slot 1024A, as seen in Fig. 16. All logical alarm notifications are centered around this network. Alarm notifications are divided into either digital or analog, which are linked to a Functional Block Diagram (FBD). Within the FBD is an SCL logical program that controls the output status of the alarm. Noting from the codes presented in Fig. 17, the alarm notification logic is divided into two parts - "Alarm status for Analog Signal" and "Alarm status for Digital Signal". Line 5 to line 20 constitutes all the bit logics for the analog signals. If the final output "Analog Alarm Status" is logically TRUE, then the notification is sent to the bit location "MS01", which in turn energizes the Buzzer, BS01 to sound. Lines 23 to 34 also show similar situation for the digital signals. The availability of the "STOP" switch, S01 provides manual OFF switch to the Buzzer. The overall digital and analog alarm status can also be observed in the Ladder logic block as seen in the block diagram.

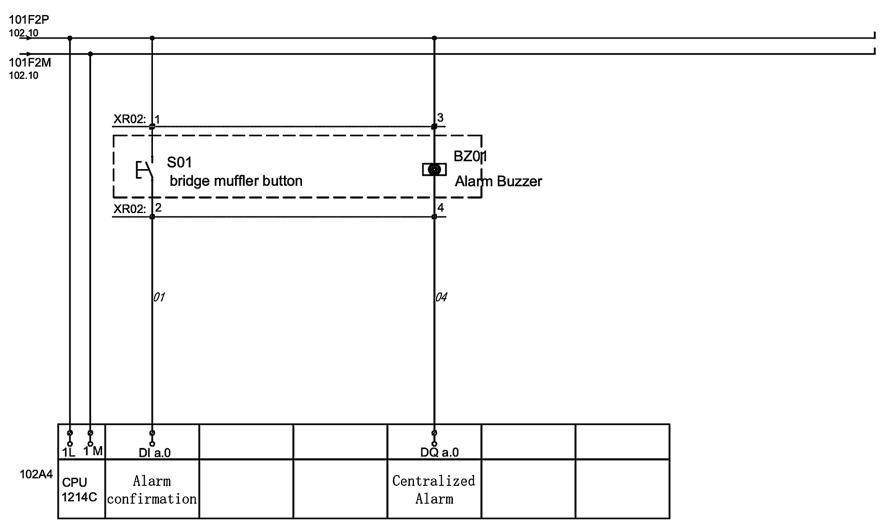

Fig. 16. Alarm network.

\section{PLC and NI Lab VIEW Communication Via OPC SERVER STATION}

Information exchange can happen in numerous ways between the programmable logic controller and NI LabVIEW. OLE for Process Control (OPC) is used in this paper as a server station to communicate between the PLC and LabVIEW. In order to do this, a device configuration was performed, out of which new channel was created. In regards, a device driver through Siemens TCP/IP Ethernet driver simulator was chosen as the communication network. Optimization configurations were performed, where the write-to-read duty cycle was set to 10 writes for every 1 read. At the same time, configurations for the handling of non-normalized floating point values were set up for the TCP/IP Ethernet driver. The Siemens S7-1200 was then added to the channel, and all tags including analog and digital tags, with their appropriate data types were added to the OPC server. The OPC tags were then connected to the LabVIEW through the creation of I/O server, that updates the connection from LabVIEW to the OPC tags every $100 \mathrm{~ms}$. Portion of the OPC tags are shown in Fig. 18.

\section{SCADA GUI DESIGN}

The Graphic User Interface (GUI) was designed in NI LabVIEW. The interface consists of five main sections -
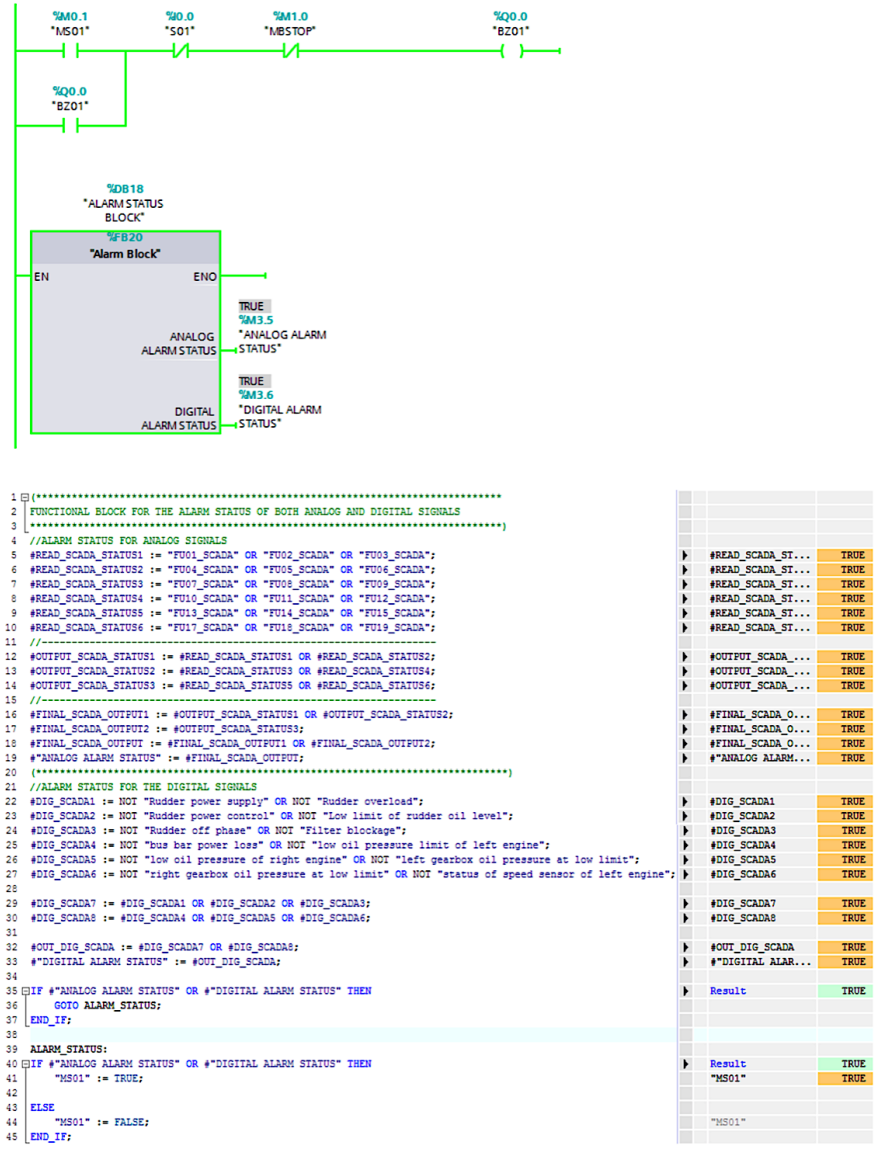

Fig. 17. Functional block diagram and SCL codes for the alarm notification.

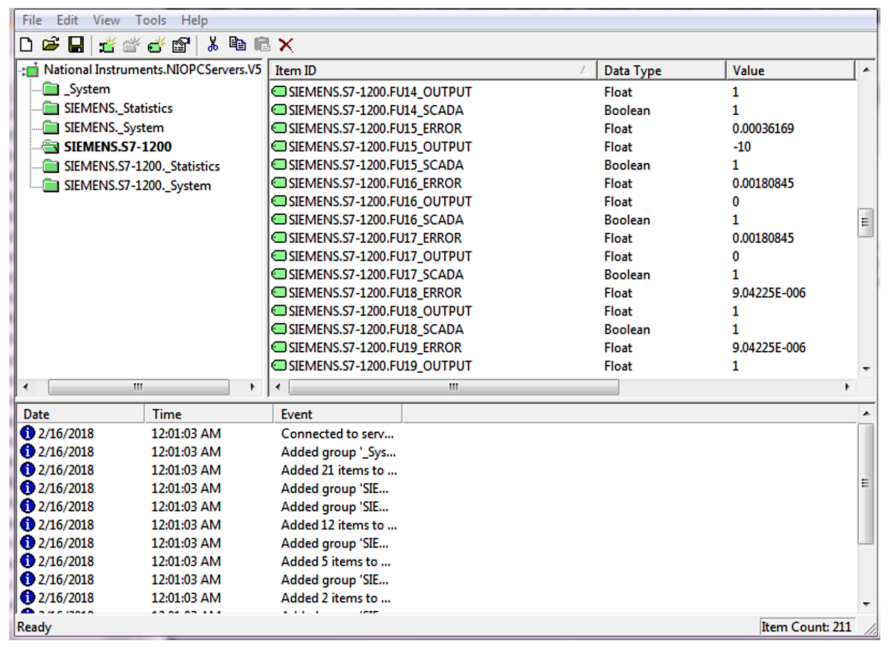

Fig. 18. OPC server configuration. 
Analog, Digital, Display, Alarm Record List, and Alarm Notification Sections. Analog and Digital Sections were put into subsections (i.e Tab 1, Tab 2, ..., Tab $\mathrm{n}$ ) to accommodate additional monitored functions. Fig. 19 shows the interface of Tab 1 of the monitored analog signals. For easier identification, functions were labeled by their fuse numbers, and separated from each other in recessed box. FUxx (for instance FU01) in the interface represents the Fuse number connected directly to the input of the monitored PLC channel. All outputs were put into limit checks, and were subjected to constant updates at $100 \mathrm{~ms}$. Display formats were set to automatic formatting, with unsigned data types capable of adapting to the source values (OPC Work Station). Signal indicator values were put into necessary precisions, were set to read-only values, and were bound through NI Shared Variable Engine. Each monitored signal was put into necessary scientific unit, and possessed alarm indicator in a form of flash button to prompt the operator of signal values that fell outside limits. Signal indicators were color coded - Red signifies "Fault" whereas Green indicates "No Fault".

Fig. 20 shows the GUI for the monitored digital signals. Digital signal identification starts with slot number and ends with a bit memory location. For instance, logical output "RUDDER POWER SUPPLY" has the label "SLOT 102A5 DI a. 0". SLOT 102A5 symbolizes the location of the signal module on the PLC Workstation, whereas a.0 indicates the bit location. Just as the case for the analog signals, digital signals possess alarm notification in a form of flash button. A red flash indicates a de-energized output, whereas a green flash signifies a working output. In each scenario, "STATUS" responds accordingly - "STATUS" displays "ON" to an energized output and "OFF" to a de-energize output.

The alarm notification section is a combination of the alarm status of all the monitored functions. "ANALOG ALARM STUTUS" responds to the status of the analog signals, whereas "DIGITAL ALARM STATUS" responds to the status of the digital signals. In each case, the flash button turns red when at least one of the signals is in the "red" state. A "green" state indicates "no fault". A written indicator that prompts the operator to "CHECK" the alarm condition can also be observed - this written indicator changes to "GOOD" when all the output statuses are in good condition. The alarm notification section provides easier fault identification. In other words, it prompts the operator of the status of the monitored functions, even before navigating through available tabs.

The "ALARM RECORD LIST" section is one of the very most important sections of the GUI design. This section records the faults and interfaces them according to date and time of the occurrence. In order to produce this, all the monitored functions are stored in a database (Microsoft Access), with their OPC tags attached. Communication is then made between the database and the LabVIEW via the OPC server. The developed program then checks the status of the faulty monitored functions, compares them with the tags in the database, and produces the list according to which fault gets detected first.

Fig. 21 shows the Display Section of the GUI. This section produces a mimic display of the functions that require quick response by the operator. In this section, monitored functions are grouped and separated from each other in a recessed box.
Display outputs consist of Temperatures, Pressures, Voltages and Current outputs. Meters are color coded and equally scaled, and labeled for easier identification by the operator. Beneath each meter is an indicator that produces precise values of the measured outputs. In many cases, creating a display section in a SCADA GUI is vital since it provides easier access to real measured values in a more simple way. In other words, it eliminates other indicator buttons. Its purpose is to serve as a platform to observe measured outputs, and not to provide details of alarm statuses. In this paper, all functions in the display section are analog functions.

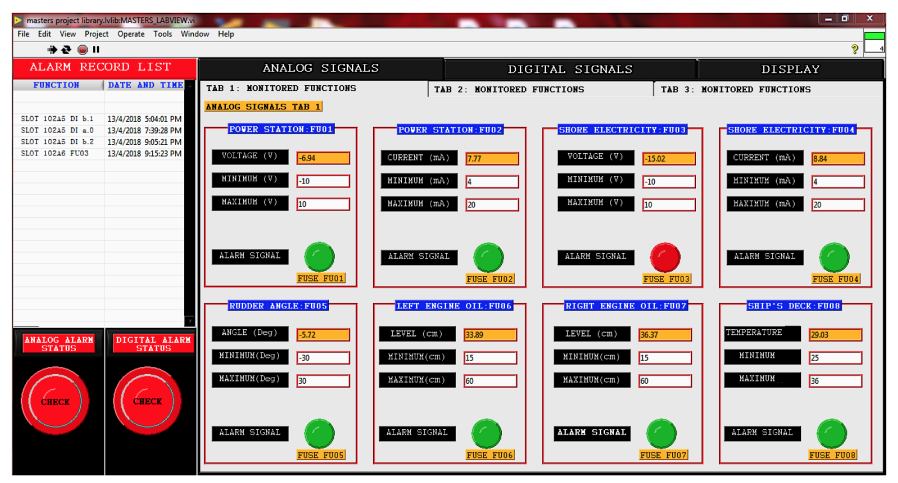

Fig. 19. Analog signals.

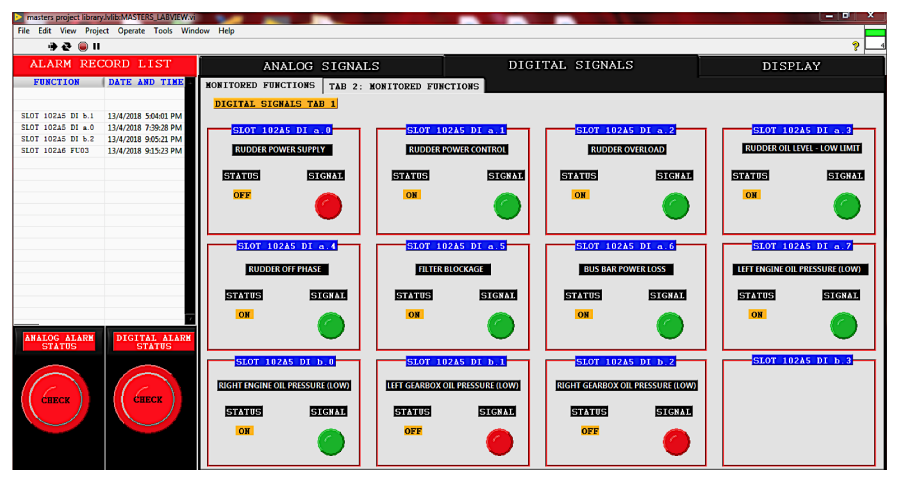

Fig. 20. Digital signals

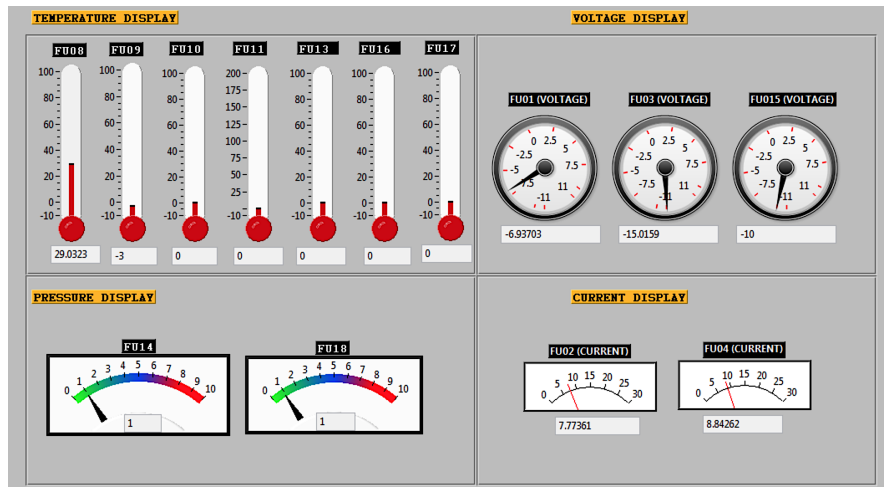

Fig. 21. GUI - Display section.

\section{CONCLUSION}

In this paper, an effective alarm design method for monitoring engine room machinery was presented. The proposed 
design proceeded through PLCs and SCADA-based systems. Reasons for such a design method were highlighted, and the programming platforms for the design were given. The strengths and weaknesses of some design methods presented in some literary works were also reported, and solutions to such problems were proposed. The designed monitoring system was tested in the shipyard for performance evaluation. The testing results proved that this design technique is reliable and effective for online engine control room device monitoring. However, as effective as it is, one peculiar limitation was encountered. This limitation is peculiar to the voltage measurements. The error control algorithm could not handle voltage spikes. A voltage spike forces the input voltage sensors to record values outside the imposed limits. This in turn produces unusual "ON" and "OFF" flicks of the voltage indicator buttons, thus interrupting the operator of the output voltage statuses. For future work, a direct pattern recognition of sensor readings for fault diagnosis is planned to be implemented. An efficient pattern recognition algorithm will help prevent the unusual $\mathrm{ON}$ and OFF behavior of indicator buttons that occur during input voltage spikes. In addition, a Web based monitoring system will be designed to monitor and share data across the internet to other off-site bases.

\section{ACKNOWLEDGMENT}

We would like to extend gratitude to Changjiang Waterway Bureau, the School of International Studies of Jiangsu University of Science and Technology, and Ministry of Education of Jiangsu province for providing the necessary financial support.

\section{REFERENCES}

[1] EEMUA, "Alarm System: A guide to design, management and procurement". The Engineering Equipment and Materials Users Association. Publication No. 191. EEMUA 45 Beech Street London EC2Y 8AD (1999).

[2] Keith Stouffer, Joe Falco, Karen Kent, Guide to supervisory control and data acquisition SCADA and industrial control systems security, National Institute of Standards and Technology, 2006. ISBN 20899- 8930.
[3] A. Lakshmi, Velammal Coll., Chennai, B. Sangeetha, A. Naveenkumar, B. Ganesh, Experimental validation of PID based cascade control system through SCADA-PLC-OPC Interface, IEEE Computer Communication and Informatics (ICCCI), (2012) pp. 1-4.

[4] Berge, Jonas. "Fieldbuses for Process Control" : Engineering, Operation, and Maintenance, ISA, 2002.

[5] M. S Zaghloul, "Online Ship Control Systems Using Supervisory Control and Data Acquisition (SCADA)". IJCSA, (2014),DOI: 10.14355/ijcsa.2014.0301.02.

[6] C. Kwon Lee, S. Hur, J.H. Shin, I. S. Koo, and J.K. Park "A basic design of Alarm System for the Future Nuclear Power Plants in Korea". Taejon, Korea. (2012).

[7] D. Sharma, A. Chaubey, A. K Gupta, "Review on Methods of Fault Detection and Protection of Induction Motor". International Journal of Computer Applications, vol 161, (2017).

[8] L. de Miguel, L. Blazquez, "Fuzzy logic-based decision-making for fault diagnosis in a DC motor", Engineering Applications of Artificial Intelligence, vol. 18, no. 4, pp. 423-450, 2005.

[9] M Iacob, G.D Andreescu, and N. Muntean. "SCADA system for a central heating and power plant". 5th International Symposium on Applied Computational Intelligence and Informatics (SACI '09), At Timisoara, Romania. DOI: 10.1109/SACI.2009.5136232.

[10] Bentley Systems, The Fundamentals of SCADA, 2004.

[11] Michael P. Ward, An architectural framework for describing supervisory control and data acquisition SCADA systems, thesis, Naval postgraduate school Monterey, California, September 2004.

[12] B.S. Jones, J.V. Earthy, and D. Gould. "Improving the design and management of alarm systems". A paper presented to the World Maritime Technology Conference, (2006).

[13] S. Da'na, "Development of a monitoring and control platform for PLCbased applications". Journal Computer Standards \& Interfaces, Vol. 30, Issue 3, pages 157-166, March 2008

[14] C. Wang, H. R. Xiao, W. G. Pan, Y. Z. Han "Design of Monitoring and Alarm System for the Ship's Engine Room", Advanced Materials Research, Vols. 268-270, pp. 1663-1668, 2011.

[15] T. Kletz, "Learning from Accidents in Industry". Butterworts, London, ISBN 0-408-02696-0, 1988, page 95.

[16] Arthur Wright, P. Gordon Newbery "Electric fuses", 3rd edition, Institution of Electrical Engineers (IET), 2004, ISBN0-86341-379-X, pp. $2-10$

[17] S7 7A 125V TD "Rejection Base Plug Fuse", Elliott Electric Supply, (2012)

[18] D. G. Fink, H.W. Beaty, "Standard Handbook for Electrical Engineers" Eleventh Edition, McGraw Hill 1978 ISBN0-07-020974-X pp.10-116 through 10-119. 